Article

\title{
The analysis of fractional-order Helmholtz equations via a novel approach
}

\author{
Pongsakorn Sunthrayuth ${ }^{1}$, Zeyad Al-Zhour ${ }^{2 *}$ and Yu-Ming Chu ${ }^{3}$ \\ 1 Department of Mathematics and Computer Science, Faculty of Science and Technology, Rajamangala \\ University of Technology Thanyaburi (RMUTT) Thanyaburi, Pathumthani, Thailand; \\ pongsakorn_su@rmutt.ac.th. \\ 2 Department of Basic Engineering Sciences, College of Engineering, Imam Abdulrahman Bin Faisal \\ university, Dammam, Saudi Arabia. \\ 3 Department of Mathematics, Huzhou University, Huzhou 313000, P. R. China; chuyuming2005@126.com. \\ * Correspondence: zalzhour@iau.edu.sa
}

Version March 5, 2021 submitted to Journal Not Specified

\begin{abstract}
This paper is related to the fractional view analysis of Helmholtz equations, using innovative analytical techniques. The fractional analysis of the proposed problems has been done in terms of Caputo-operator sense. In the current methodology, first, we applied the $\rho$-Laplace transform to the targeted problem. The iterative method is then implemented to obtain the series form solution. After using the inverse transform of the $\rho$-Laplace, the desire analytical solution is achieved. The suggested procedure are verified through specific examples of the fractional Helmholtz equations. The present method is found to be an effective technique having a closed resemblance with the actual solutions. The proposed technique have less computational cost and a higher rate of convergence. The suggested methods are therefore very useful to solve other systems of fractional order problems.
\end{abstract}

Keywords: New Iterative method, $\rho$-Laplace transform, Fractional-order Helmholtz equations, Caputo operator.

\section{Introduction}

In general, fractional calculus has been a significant field of applied mathematics in the last several centuries. Good results than classical derivatives are obtained by modeling real phenomena with fractional derivative and fractional integral. In the modeling of certain physical phenomena, some important applications can be traced, visco-elasticity damping, especially signal processing, electronics, genetic algorithms, communication, transport systems, robotics, biology, chemistry, physics and finance. In the field of fractional calculus, several researchers are focusing on several significant discoveries and contributions $[13,14]$. For most observers and scholars, fractional calculus is an important research field due to its interesting applications, and the investigation of fractional-order partial differential equations (PDEs) has attracted specific attention from various researchers. In light of this, a number of approaches have been used to solve different linear and nonlinear fractional PDEs. For instance, for solving the time-fractional and anomalous mobile-immobile solution transport process, the local meshless approach is used $[5-8,15,16]$.

The Helmholtz equation or reduced wave equation is an elliptical partial differential equation (PDE) derived directly from the wave model. Helmholtz equation is a PDE that signifies time-independent mechanical growth in the universe. The Helmholtz equation is one of the essential applied mathematics and physics equations. The Helmholtz equation results, which are usually created from the separation of variables, address important science phenomena. Equations occur in a number of phenomena, such as vibrating lines, electromagnetic Waves in fluids, walls, plates, Nuclear plants, acoustics, magnetic 
fields and the Lamb equation in geoscience. Consider a multi dimensional nonhomogenous isotropic medium whose velocity is $c$ in euclidean space $[9,10]$. The wave outcome is $\mu(\xi, \zeta)$ consistent to the harmonic origin $\phi(\xi, \zeta)$ vibrating at the identified fixed frequency $\omega>0$ satisfies the Helmholtz equation for the defined field $R$ :

$$
\frac{\partial^{2} \mu(\xi, \zeta)}{\partial \xi^{2}}+\frac{\partial^{2} \mu(\xi, \zeta)}{\partial \zeta^{2}}+\lambda \mu(\xi, \zeta)=-\phi(\xi, \zeta),
$$

Where $\mu(\xi, \zeta)$ is an appropriately differentiable function at the boundary of $R$, and $\phi(\xi, \zeta)$ is a specified function, $\lambda>0$ is a constant value, and $\sqrt{\lambda}=\frac{\omega}{c}$ is a wave number with a wave length of $\frac{2 \pi}{\sqrt{\lambda}}$. If $\phi(\xi, \zeta)=0$ is necessary, then equation (1) The Helmholtz equation is homogeneous. Most equations related to steady-state oscillations (acoustic, electromagnetic, thermoelectric, hydraulic) lead to a two-dimensions Helmholtz equation, if the positive sign (in front of the $\lambda$ term) is improved to a negative sign, then this problem describes mass transport of processes with first-order volume chemical mixtures. For example, in linear acoustics, $\phi(\xi, \zeta)$ can reflect a disturbance of the reference state pressure (Thompson and Pinsky, 1995). Conservation equations, which are also transformed into Helmholtz equations, often have a wide variety of applications in many physical problems, such as fluid constrained or shear viscosity streams inside thermophysical walls [9-12]. In current years, numerous analytical and numerical techniques have been applied to solve fractional-order Helmholtz equations, such as He's homotopy perturbation method [13], Laplace variational iteration method [14], Reduced Differential Transform method [15], q-homotopy analysis transform method [16], spectral method [17] and He's variational iteration method [18].

In recent years, fractional-order calculus has emerged as a potential tool in various science and technology domains such as possible theory, control model, viscoelasticity, fluid dynamic traffic, electromagnetic theory, electric technology, business mathematics and bioengineering. The real-world processes we deal with are generally of fractional order. The diffusion of heat into a semi-infinite solid where the heat movement is equal to the fractional-derivative of temperature is a case in point of a fractional-order instrument.

Recently, Abdeljawad and Fahd [19] introduced the Laplace transformation of the fractional-order Caputo derivatives. We suggested a new iterative technique with $\rho$-Laplace transformation for investigation of fractional-order ordinary and pratial differential equations with fractional-order Caputo derivative. We apply this novel suggested technique for solving many fractional-order differential equations such as linear and nonlinear diffusion equation, fractional-order Zakherov-Kuznetsov equation and Fokker-Planck equations. We analyzed the impact of $\alpha$ and $\rho$ in the process.

\section{Basic definitions}

In this section, the generalized fractional derivative, the generalized fractional integral, the Mittag-Lefller function the $\rho$-Laplace transform have been discussed.

\subsection{Definition}

The generalized fractional-order integral $\alpha$ of a continuous function $f:[0,+\infty] \rightarrow R$ is expressed as[19]

$$
\left(I^{\alpha, \rho} f\right)(\zeta)=\frac{1}{\Gamma(\alpha)} \int_{0}^{\zeta}\left(\frac{\zeta^{\rho}-s^{\rho}}{\rho}\right)^{\alpha-1} \frac{f(s) d s}{s^{1-\rho}},
$$

where denotes the gamma function is $\Gamma, \rho>0, \zeta>0$ and $0<\alpha<1$. 


\subsection{Definition}

The generalized fractional-order derivative of $\alpha$ of a continuous function $f:[0,+\infty] \rightarrow R$ is given as [19].

$$
\left(D^{\gamma, \rho} f\right)(\zeta)=\left(I^{1-\alpha, \rho} f\right)(\zeta)=\frac{1}{\Gamma(1-\alpha)}\left(\frac{d}{d \zeta}\right) \int_{0}^{\zeta}\left(\frac{\zeta^{\rho}-s^{\rho}}{\rho}\right)^{-\alpha-1} \frac{f(s) d s}{s^{1-\rho}} .
$$

where define the gamma function $\Gamma, \rho>0, \zeta>0$ and $0<\alpha<1$.

\subsection{Definition}

The Caputo fractional-order derivative $\alpha$ of a continuous function $f:[0,+\infty] \rightarrow R$ is expressed as [19]

$$
\left(D^{\gamma, \rho} f\right)(\zeta)=\frac{1}{\Gamma(1-\alpha)}\left(\frac{d}{d \zeta}\right) \int_{0}^{\zeta}\left(\frac{\zeta^{\rho}-s^{\rho}}{\rho}\right)^{-\alpha-1} \gamma^{n} \frac{f(s) d s}{s^{1-\rho}}
$$

where $\rho>0, \zeta>0, \gamma=\zeta^{1-\gamma} \frac{d}{d \zeta}$ and $0<\alpha<1$.

\subsection{Definition}

The $\rho$-Laplace transformation of a continuous function $f:[0,+\infty] \rightarrow R$ is given as [19]

$$
L_{\rho}\{f(\zeta)\}(s)=\int_{0}^{\infty} e^{-s \frac{\zeta^{\rho}}{\rho}} f(\zeta) \frac{d \zeta}{\zeta^{1-\rho}}
$$

The Caputo generalized fractional-order $\rho$-Laplace transform derivative of a continuous function $f$ is defined by [19].

$$
L_{\rho}\left\{D^{\alpha, \rho} f(\zeta)\right\}(s)=s^{\alpha} L_{\rho}\{f(\zeta)\}-\sum_{k=0}^{n-1} s^{\alpha-k-1}\left(I^{\alpha, \rho} \gamma^{n} f\right)(0)
$$

\subsection{Definition}

The Mittag-Leffler generalized function is given by

$$
E_{\alpha, \rho}(z)=\sum_{k=0}^{\infty} \frac{z^{\alpha}}{\Gamma(\alpha k+\beta)}
$$

where $\alpha>0, \beta>0$ and $E_{\alpha}(z)=E_{\alpha, 1}(z)$.

\section{The new iterative technique general methodology with $\rho$-Laplace transformation}

Consider the functional equation

$$
\varphi=f+L(\varphi)+N(\varphi)
$$

where the function denote by $f$, linear and nonlinear function of $\varphi$ show by $L$ and $N$, respectively. Let us consider solution of (3) is of the form

$$
\begin{aligned}
\varphi_{0} & =f, \\
\varphi_{1} & =L\left(\varphi_{0}\right)+N\left(\varphi_{0}\right), \\
\varphi_{m+1} & =L\left(\varphi_{m}\right)+\left(N\left(\sum_{k=0}^{\infty} \varphi_{m}(r)\right)-N\left(\sum_{k=0}^{m-1} \varphi_{k}(r)\right)\right),
\end{aligned}
$$


where $m=1,2,3 \cdots$,

$$
u=\sum_{k=0}^{\infty} \varphi_{k}
$$

Now, linear and nonlinear form can be define as [28]:

$$
\begin{gathered}
L(\varphi)=L\left(\sum_{k=0}^{\infty} \varphi_{k}\right)=\left(\sum_{k=0}^{\infty} L\left(\varphi_{k}\right)\right), \\
N(\varphi)=N\left(\sum_{k=0}^{\infty} \varphi_{k}\right)=N\left(\varphi_{0}\right)+\sum_{k=1}^{\infty}\left\{N\left(\sum_{j=0}^{k} \varphi_{j}\right)-N\left(\sum_{j=0}^{k-1} \varphi_{j}\right)\right\} .
\end{gathered}
$$

We achieve the result of (3) as

$$
\varphi=f+\sum_{k=0}^{\infty} L\left(\varphi_{k}\right)+N\left(\varphi_{0}\right)+\sum_{k=1}^{\infty}\left\{N\left(\sum_{j=0}^{k} \varphi_{j}\right)-N\left(\sum_{j=0}^{k-1} \varphi_{j}\right)\right\}
$$

Consider the initial value fractional-order equation with Caputo derivative as

$$
D^{\alpha, \rho} \varphi(\xi, \zeta)+N \varphi(\xi, \zeta)=f(\xi, \zeta)
$$

with initial condition $\varphi(\xi, 0)=g(\xi)$, where $\mathrm{N}$ is a nonlinear function.

Now on both sides using $\rho$-Laplace transformation of Eq. (8),

we get

$$
L_{\rho}\left(D^{\alpha, \rho} \varphi(\xi, \zeta)+N u(\xi, \zeta)\right)=L_{\rho}(f(\xi, \zeta))
$$

applying (2), we achieve

$$
s^{\alpha} \varphi(\xi, s)-s^{\alpha-1} \varphi(\xi, 0)=L_{\rho}(f(\xi, \zeta))-L_{\rho}(N \varphi(\xi, \zeta)) .
$$

Simplify (10), we get

$$
\varphi(\xi, s)=\frac{\varphi_{0}(\xi, s)}{s}+s^{-\alpha} L_{\rho}(f(\xi, \zeta))-s^{-\alpha} L_{\rho}(N \varphi(\xi, \zeta)) .
$$

Taking on both side inverse Laplace transformation of (12), we get

$$
\varphi(\xi, \zeta)=\varphi_{0}(\xi, s)+L_{\rho}^{-1}\left(s^{-\alpha} L_{\rho}(f(\xi, \zeta))\right)-L_{\rho}^{-1}\left(s^{-\alpha} L_{\rho}(N u(\xi, \zeta))\right) .
$$

Using new iterative technique, we have

$$
\begin{aligned}
f & =\varphi(x, 0), \\
L(\varphi) & =L_{\rho}^{-1}\left(s^{-\alpha} L_{\rho}(f(\xi, \zeta))\right), \\
N(\varphi) & =-L_{\rho}^{-1}\left(s^{-\alpha} L_{\rho}(N \varphi(\xi, \zeta))\right) .
\end{aligned}
$$

The recurrence relation is given as,

$$
\begin{aligned}
\varphi_{0} & =f, \\
\varphi_{1} & =L\left(\varphi_{0}\right)+N\left(\varphi_{0}\right), \\
\varphi_{m+1} & =L\left(\varphi_{m}\right)+\left(N\left(\sum_{k=0}^{\infty} \varphi_{m}(r)\right)-N\left(\sum_{k=0}^{m-1} \varphi_{k}(r)\right)\right),
\end{aligned}
$$


where $m=1,2,3 \cdots$, Hence, the result of $(8)$ is of the form,

$$
\sum_{k=0}^{\infty} \varphi_{k}=f+L\left(\sum_{k=0}^{\infty} \varphi_{k}\right)+N\left(\sum_{k=0}^{\infty} \varphi_{k}\right)
$$

\section{Application}

Example 1. Consider the fractional Helmholtz equation is given as

$$
D_{\xi}^{\alpha, \rho} \varphi(\xi, \zeta)+\frac{\partial^{2} \varphi(\xi, \zeta)}{\partial \zeta^{2}}-\varphi(\xi, \zeta)=0, \quad 1<\alpha \leq 2
$$

with $\varphi(0, \zeta)=\zeta$ and $\varphi_{\xi}(0, \zeta)=0$. Using on both side $\rho$-Laplace transformation of Eq.(34), we get

$$
\varphi(\xi, s)=\frac{L_{\rho}\left(\varphi_{\xi \xi}\right)+s^{\alpha-1} \varphi(0, \zeta)+s^{\alpha-2} \varphi_{\xi}(0, \zeta)}{s^{\alpha}},
$$

from which,

$$
\varphi(\xi, \zeta)=\frac{\varphi(0, \zeta)}{s}-\frac{L_{\rho}\left\{\frac{\partial^{2} \varphi(\xi, \zeta)}{\partial \zeta^{2}}-\varphi(\xi, \zeta)\right\}}{s^{\alpha}}
$$

Implement on both sides inverse $\rho$-Laplace transformation of (36), we get

$$
\varphi(\xi, \zeta)=\zeta-L_{\rho}^{-1}\left[\frac{L_{\rho}\left\{\frac{\partial^{2} \varphi(\xi, \zeta)}{\partial \zeta^{2}}-\varphi(\xi, \zeta)\right\}}{s^{\alpha}}\right]
$$

Applying technique of new suggested method, we get

$$
\begin{gathered}
f=\varphi_{0}=\zeta, \\
\varphi_{1}(\xi, \zeta)=-L_{\rho}^{-1}\left[\frac{L_{\rho}\left\{\frac{\partial^{2} \varphi_{0}(\xi, \zeta)}{\partial \zeta^{2}}-\varphi_{0}(\xi, \zeta)\right\}}{s^{\alpha}}\right]=\frac{\zeta}{\Gamma(1+\alpha)}\left(\frac{\xi^{\rho}}{\rho}\right)^{\alpha}, \\
\varphi_{2}(\xi, \zeta)=-L_{\rho}^{-1}\left[\frac{L_{\rho}\left\{\frac{\partial^{2} \varphi_{1}(\xi, \zeta)}{\partial \zeta^{2}}-\varphi_{1}(\xi, \zeta)\right\}}{s^{\alpha}}\right]=\frac{\zeta}{\Gamma(1+2 \alpha)}\left(\frac{\xi^{\rho}}{\rho}\right)^{2 \alpha}, \\
\varphi_{3}(\xi, \zeta)=-L_{\rho}^{-1}\left[\frac{L_{\rho}\left\{\frac{\partial^{2} \varphi_{2}(\xi, \zeta)}{\partial \zeta^{2}}-\varphi_{2}(\xi, \zeta)\right\}}{s^{\alpha}}\right]=\frac{\zeta}{\Gamma(1+3 \alpha)}\left(\frac{\xi^{\rho}}{\rho}\right)^{3 \alpha},
\end{gathered}
$$

Hence, the analytical result of Eq. (34) is given in terms of Mittag-Leffler function as:

$$
\begin{aligned}
& \varphi(\xi, \zeta)=\varphi_{0}+\varphi_{1}+\varphi_{2}+\varphi_{3}+\cdots \\
& \varphi(\xi, \zeta)=\zeta+\frac{\zeta}{\Gamma(1+\alpha)}\left(\frac{\xi^{\rho}}{\rho}\right)^{\alpha}+\frac{\zeta}{\Gamma(1+2 \alpha)}\left(\frac{\xi^{\rho}}{\rho}\right)^{2 \alpha}+\frac{\zeta}{\Gamma(1+3 \alpha)}\left(\frac{\xi^{\rho}}{\rho}\right)^{3 \alpha}+\cdots
\end{aligned}
$$

The exact solution of the problem 1 when $\alpha=2$,

$$
\varphi(\xi, \zeta)=\zeta \cosh \xi
$$


In the same producer the result of $\zeta$-space can be determined through ITM as:

$$
D_{\zeta}^{\alpha, \rho} \varphi(\xi, \zeta)+\frac{\partial^{2} \varphi(\xi, \zeta)}{\partial x^{2}}-\varphi(\xi, \zeta)=0
$$

with initial condition

$$
\varphi(\xi, 0)=\xi
$$

Thus the solution of the above (31) is obtained as

$$
\varphi(\xi, \zeta)=\xi+\frac{\xi}{\Gamma(1+\alpha)}\left(\frac{\zeta^{\rho}}{\rho}\right)^{\alpha}+\frac{\xi}{\Gamma(1+2 \alpha)}\left(\frac{\zeta^{\rho}}{\rho}\right)^{2 \alpha}+\frac{\xi}{\Gamma(1+3 \alpha)}\left(\frac{\zeta^{\rho}}{\rho}\right)^{3 \alpha}+\cdots
$$

in the case when $\alpha=2$, then the result through ITM is

$$
\varphi(\xi, \zeta)=\xi \cosh \zeta
$$

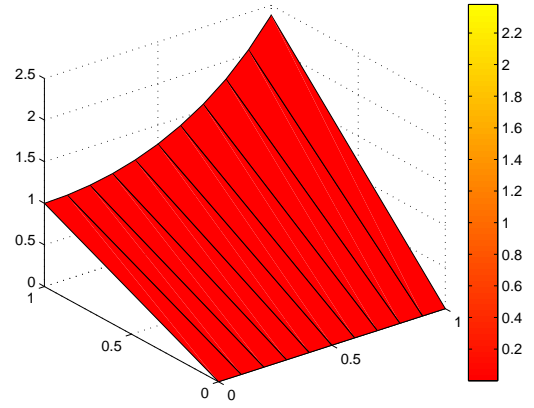

(a) The exact solution plot of example 1.

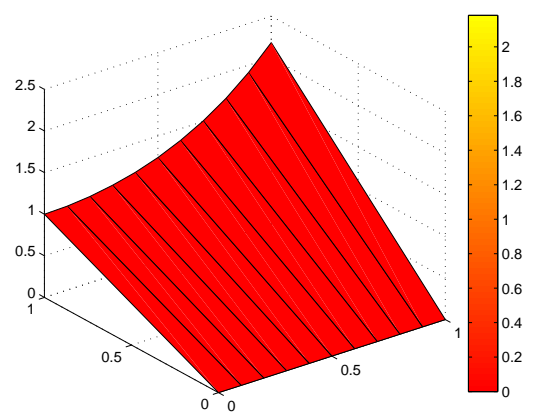

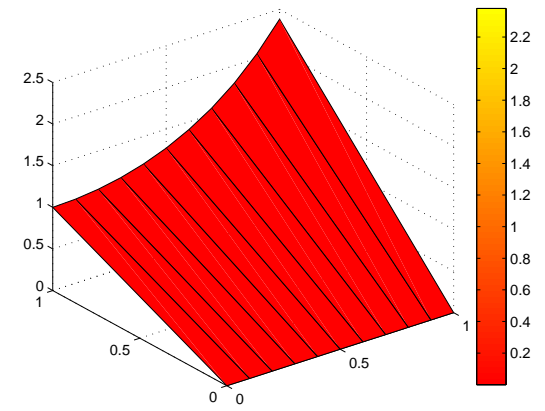

(b) ITM graph of example 1.

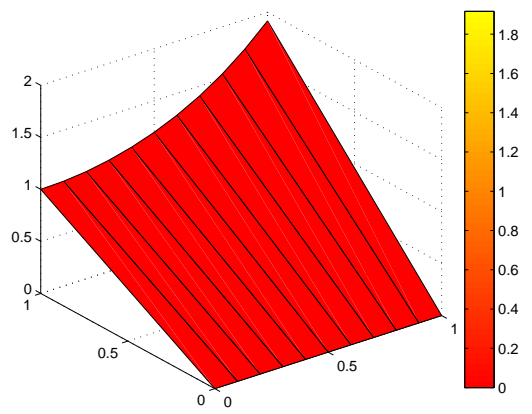

(c) ITM graph at fractional-order $\gamma=1.8$ of example 1. (d) ITM graph at fractional-order $\gamma=1.6$ of example 1.

Figure 1

Example 2. Consider the fractional Helmholtz equation is given as

$$
D_{\xi}^{\alpha, \rho} \varphi(\xi, \zeta)+\frac{\partial^{2} \varphi(\xi, \zeta)}{\partial \zeta^{2}}+5 \varphi(\xi, \zeta)=0, \quad 1<\alpha \leq 2,
$$

with $\varphi(0, \zeta)=\zeta$ and $\varphi_{\zeta}(0, \zeta)=0$. Using on both sides $\rho$-Laplace transformation of Eq.(34), we achieve

$$
\varphi(\xi, s)=\frac{L_{\rho}\left(\varphi_{\xi \xi}\right)+s^{\alpha-1} \varphi(0, \zeta)+s^{\alpha-2} \varphi_{\xi}(0, \zeta)}{s^{\alpha}},
$$


from which,

$$
\varphi(\xi, \zeta)=\frac{\varphi(0, \zeta)}{s}+\frac{\varphi_{\xi}(0, \zeta)}{s^{2}}-\frac{L_{\rho}\left\{\frac{\partial^{2} \varphi(\xi, \zeta)}{\partial \zeta^{2}}+5 \varphi(\xi, \zeta)\right\}}{s^{\alpha}}
$$

Using on both sides inverse $\rho$-Laplace transformation of Eq. (36), we get

$$
\varphi(\xi, \zeta)=\zeta-L_{\rho}^{-1}\left[\frac{L_{\rho}\left\{\frac{\partial^{2} \varphi(\xi, \zeta)}{\partial \zeta^{2}}+5 \varphi(\xi, \zeta)\right\}}{s^{\alpha}}\right]
$$

Applying technique of new suggested method, we have

$$
\begin{gathered}
f=\varphi_{0}=\zeta, \\
\varphi_{1}(\xi, \zeta)=-L_{\rho}^{-1}\left[\frac{L_{\rho}\left\{\frac{\partial^{2} \varphi_{0}(\xi, \zeta)}{\partial \zeta^{2}}+5 \varphi_{0}(\xi, \zeta)\right\}}{s^{\alpha}}\right]=\frac{-5 \zeta}{\Gamma(1+\alpha)}\left(\frac{\xi^{\rho}}{\rho}\right)^{\alpha}, \\
\varphi_{2}(\xi, \zeta)=-L_{\rho}^{-1}\left[\frac{L_{\rho}\left\{\frac{\partial^{2} \varphi_{1}(\xi, \zeta)}{\partial \zeta^{2}}+5 \varphi_{1}(\xi, \zeta)\right\}}{s^{\alpha}}\right]=\frac{25 \zeta}{\Gamma(1+2 \alpha)}\left(\frac{\xi^{\rho}}{\rho}\right)^{2 \alpha}, \\
\varphi_{3}(\xi, \zeta)=-L_{\rho}^{-1}\left[\frac{L_{\rho}\left\{\frac{\partial^{2} \varphi_{2}(\xi, \zeta)}{\partial \zeta^{2}}+5 \varphi_{2}(\xi, \zeta)\right\}}{s^{\alpha}}\right]=\frac{-125 \zeta}{\Gamma(1+3 \alpha)}\left(\frac{\xi^{\rho}}{\rho}\right)^{3 \alpha},
\end{gathered}
$$

Hence, the approximate result of Eq. (34) is given in recursive of Mittag-Leffler function as:

$$
\begin{aligned}
& u(\xi, \zeta)=\varphi_{0}+\varphi_{1}+\varphi_{2}+\varphi_{3}+\cdots \\
& u(\xi, \zeta)=\zeta-5 \frac{\zeta}{\Gamma(1+\alpha)}\left(\frac{\xi^{\rho}}{\rho}\right)^{\alpha}+25 \frac{\zeta}{\Gamma(1+2 \alpha)}\left(\frac{\xi^{\rho}}{\rho}\right)^{2 \alpha}-125 \frac{\zeta}{\Gamma(1+3 \alpha)}\left(\frac{\xi^{\rho}}{\rho}\right)^{3 \alpha}+\cdots .
\end{aligned}
$$

The exact solution of the problem 1 when $\alpha=2$,

$$
\varphi(\xi, \zeta)=\zeta \cos \sqrt{5} \xi
$$

In the same producer the result of $y$-space can be calculated through ITM as:

$$
D_{\zeta}^{\alpha, \rho} \varphi(\xi, \zeta)+\frac{\partial^{2} \varphi(\xi, \zeta)}{\partial x^{2}}+5 \varphi(\xi, \zeta)=0
$$

with the initial condition

$$
\varphi(\xi, 0)=\xi, \varphi_{\zeta}(\xi, 0)=0 .
$$

Thus the solution of the above (31) is obtain by

$$
\varphi(\xi, \zeta)=\xi-5 \frac{\xi}{\Gamma(1+\alpha)}\left(\frac{\zeta^{\rho}}{\rho}\right)^{\alpha}+25 \frac{\xi}{\Gamma(1+2 \alpha)}\left(\frac{\zeta^{\rho}}{\rho}\right)^{2 \alpha}-125 \frac{\xi}{\Gamma(1+3 \alpha)}\left(\frac{\zeta^{\rho}}{\rho}\right)^{3 \alpha}+\cdots
$$

in the case when $\alpha=2$, then the result through ITM is

$$
\varphi(\xi, \zeta)=\xi \cos \sqrt{5} \zeta
$$




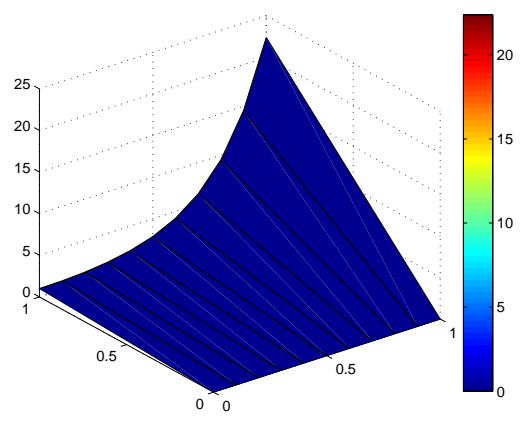

(a) The exact solution plot of example 2.

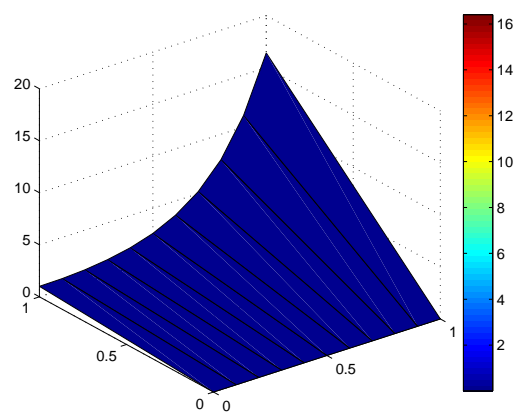

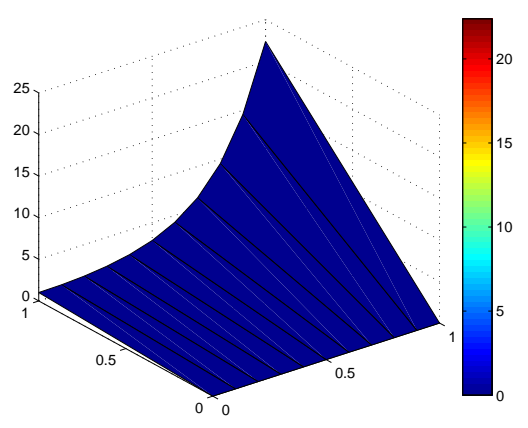

(b) ITM graph of example 2.

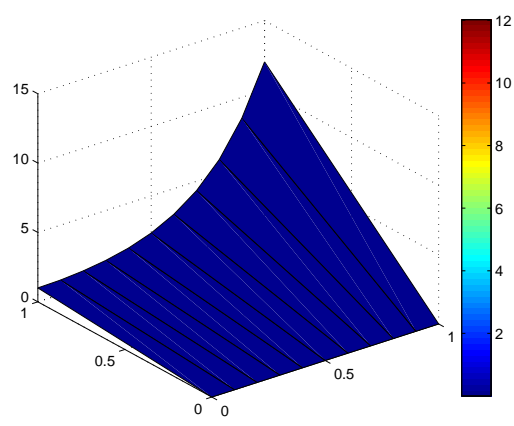

(c) ITM graph at fractional-order $\gamma=1.8$ of example 2. (d) ITM graph at fractional-order $\gamma=1.6$ of example 2 .

Figure 2

Example 3. Consider the fractional Helmholtz equations

$$
D_{\xi}^{\alpha, \rho} \varphi(\xi, \zeta)+\frac{\partial^{2} \varphi(\xi, \zeta)}{\partial \zeta^{2}}-\varphi(\xi, \zeta)=0, \quad 1<\alpha \leq 2,
$$

with $\varphi(0, \zeta)=\zeta$ and $\varphi_{\xi}(0, \zeta)=\zeta+\cosh (\zeta)$. Using on both side $\rho$-Laplace transform of Eq.(34), we achieve

$$
\varphi(\xi, s)=\frac{L_{\rho}\left(\varphi_{\xi \xi}\right)+s^{\alpha-1} \varphi(0, \zeta)+s^{\alpha-2} \varphi_{\xi}(0, \zeta)}{s^{\alpha}}
$$

from which,

$$
\varphi(\xi, \zeta)=\frac{\varphi(0, \zeta)}{s}+\frac{\varphi_{\xi}(0, \zeta)}{s^{2}}-\frac{L_{\rho}\left\{\frac{\partial^{2} \varphi(\xi, \zeta)}{\partial \zeta^{2}}-\varphi(\xi, \zeta)\right\}}{s^{\alpha}} .
$$

Implement on both side inverse $\rho$-Laplace transformation of (36), we get

$$
\varphi(\xi, \zeta)=\zeta(1+\xi)+\xi \cosh (\zeta)-L_{\rho}^{-1}\left[\frac{L_{\rho}\left\{\frac{\partial^{2} \varphi(\xi, \zeta)}{\partial \zeta^{2}}-\varphi(\xi, \zeta)\right\}}{s^{\alpha}}\right]
$$


Applying technique of new suggested method, we have

$$
\begin{gathered}
f=\varphi_{0}=\zeta(1+\xi)+\xi \cosh (\zeta), \\
\varphi_{1}(\xi, \zeta)=-L_{\rho}^{-1}\left[\frac{L_{\rho}\left\{\frac{\partial^{2} \varphi_{0}(\xi, \zeta)}{\partial \zeta^{2}}-\varphi_{0}(\xi, \zeta)\right\}}{s^{\alpha}}\right]=\zeta\left(\frac{\left(\frac{\xi^{\rho}}{\rho}\right)^{2 \alpha}}{\Gamma(1+2 \alpha)}+\frac{\left(\frac{\xi^{\rho}}{\rho}\right)^{3 \alpha}}{\Gamma(1+3 \alpha)}\right), \\
\varphi_{2}(\xi, \zeta)=-L_{\rho}^{-1}\left[\frac{L_{\rho}\left\{\frac{\partial^{2} \varphi_{1}(\xi, \zeta)}{\partial \zeta^{2}}-\varphi_{1}(\xi, \zeta)\right\}}{s^{\alpha}}\right]=\zeta\left(\frac{\left(\frac{\xi^{\rho}}{\rho}\right)^{4 \alpha}}{\Gamma(1+4 \alpha)}+\frac{\left(\frac{\xi^{\rho}}{\rho}\right)^{5 \alpha}}{\Gamma(1+5 \alpha)}\right), \\
\varphi_{3}(\xi, \zeta)=-L_{\rho}^{-1}\left[\frac{L_{\rho}\left\{\frac{\partial^{2} \varphi_{2}(\xi, \zeta)}{\partial \zeta^{2}}-\varphi_{2}(\xi, \zeta)\right\}}{s^{\alpha}}\right]=\zeta\left(\frac{\left(\frac{\xi^{\rho}}{\rho}\right)^{6 \alpha}}{\Gamma(1+6 \alpha)}+\frac{\left(\frac{\xi^{\rho}}{\rho}\right)^{7 \alpha}}{\Gamma(1+7 \alpha)}\right),
\end{gathered}
$$

Hence, the approximate result of (34) is given in recursive of Mittag-Leffler function as:

$$
\begin{aligned}
\varphi(\xi, \zeta) & =\varphi_{0}+\varphi_{1}+\varphi_{2}+\varphi_{3}+\cdots \\
\varphi(\xi, \zeta) & =\xi \cosh (\zeta)+\zeta(1+\xi)+\frac{\zeta}{\Gamma(1+\alpha)}\left(\frac{\xi^{\rho}}{\rho}\right)^{\alpha}+\frac{\zeta}{\Gamma(1+2 \alpha)}\left(\frac{\xi^{\rho}}{\rho}\right)^{2 \alpha}+\frac{\zeta}{\Gamma(1+3 \alpha)}\left(\frac{\xi^{\rho}}{\rho}\right)^{3 \alpha} \\
& +\frac{\zeta}{\Gamma(1+4 \alpha)}\left(\frac{\xi^{\rho}}{\rho}\right)^{4 \alpha}+\frac{\zeta}{\Gamma(1+5 \alpha)}\left(\frac{\xi^{\rho}}{\rho}\right)^{5 \alpha}+\frac{\zeta}{\Gamma(1+6 \alpha)}\left(\frac{\xi^{\rho}}{\rho}\right)^{6 \alpha}+\cdots
\end{aligned}
$$

The exact solution of the problem when $\alpha=2$,

$$
\varphi(\xi, \zeta)=\zeta e^{\xi}+\xi \cosh \zeta
$$

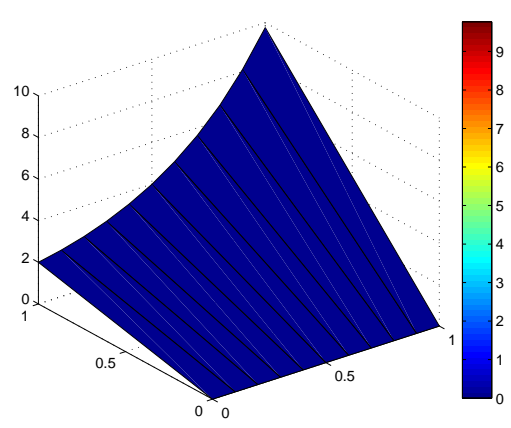

(a) The exact solution plot of example 3.

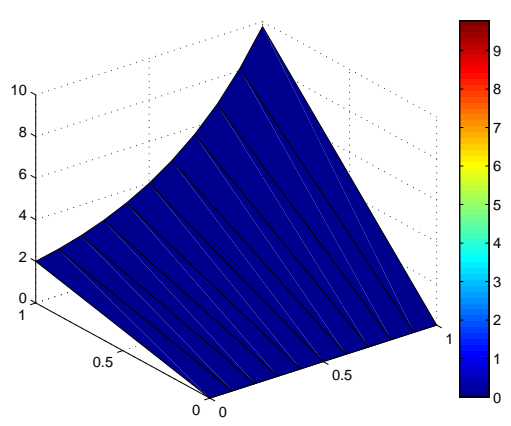

(b) ITM graph of example 3. 

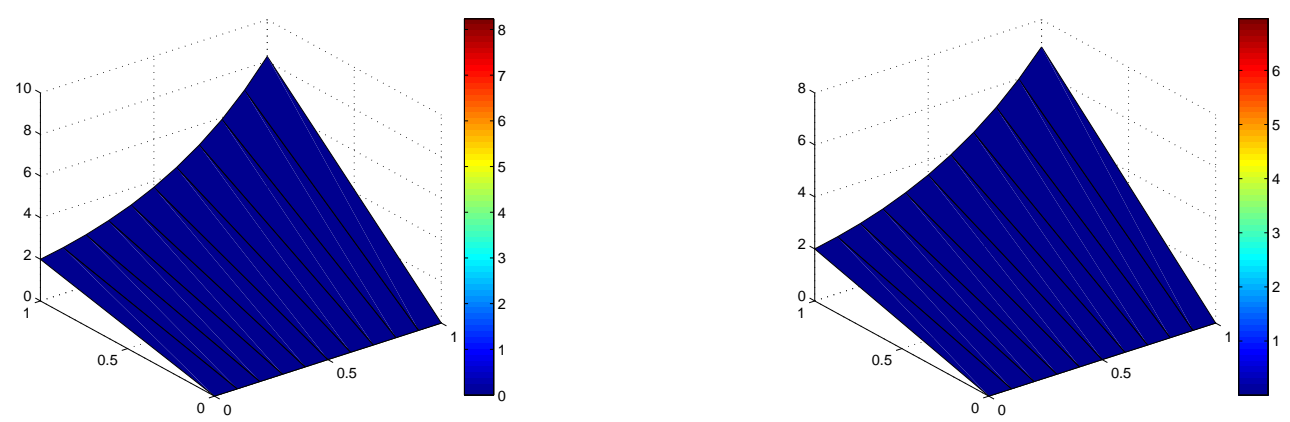

(c) ITM graph at fractional-order $\gamma=1.8$ of example 3. (d) ITM graph at fractional-order $\gamma=1.6$ of example 3 .

Figure 3

\section{Results Discussion}

Fig. 1, represents the solution-graphs of example 1 at fractional-orders $\alpha=1$, which are represented by left and right sub-graphs of Fig. 1, respectively. Fig. 1 is plotted to show the fractional-order solutions of example 1 at fractional-orders , 0.8 and 0.6 by the left and right sub-graphs, respectively. In Fig. 2, the graph of integer-order solutions of example 2 is presented and confirmed various dynamical behaviours of the physical phenomenon, which is modelled by the equation given in example 2. In Fig. 2, two subgraphs (c)and (d) left and right represented the solution of example 2 at fractional-order 0.8 and 0.6, respectively. In Fig. 3, the solutions of example 3 at integer-order are discussed. In Fig. 3, the graph of fractional-orders solutions at 0.8 and 0.6 is displaced and the systematic behaviour of the solution is observed. In both cases, we observed the correct behaviour of the solutions, which confirmed the validity of the suggested method.

\section{Conclusion}

In the present article, iterative transform method is applied for the solution of fractional-order Helmholtz equations. The graphical representations of the derived results have been done. These representation of the obtained results have clearly confirmed the higher accuracy of the suggested method. The solutions are obtained for fractional systems which are closely related to their actual solutions. The convergence of fractional solutions to integer-order solution have been shown. The less calculations and higher accuracy are the valuable themes of the present method. The researchers are then modified it to solve other fractional-order linear and nonlinear partial differential equations.

\section{References}

1. A. Carpinteri, F. Mainardi, Fractals and Fractional Calculus in Continuum Mechanics, Springer Verlag, Wien, New York, 1997.

2. K.S. Miller, B. Ross, An Introduction to the Fractional Calculus and Fractional Differential Equations, Wiley, New York, 1993.

3. K.B. Oldham, J. Spanier, The Fractional Calculus, Academic Press, New York, 1974.

4. I. Podlubny, Fractional Differential Equations, Academic Press, San Diego, 1999.

5. Ahmad, H., Akgul, A., Khan, T.A., Stanimirovic, P.S. and Chu, Y.M., 2020. New perspective on the conventional solutions of the nonlinear time-fractional partial differential equations. Complexity, 2020.

6. Qu, H., She, Z. and Liu, X., 2020. Homotopy Analysis Method for Three Types of Fractional Partial Differential Equations. Complexity, 2020.

7. Hattaf, K. and Yousfi, N., 2020. Global stability for fractional diffusion equations in biological systems. Complexity, 2020.

8. Agarwal, P., Baltaeva, U. and Alikulov, Y., 2020. Solvability of the boundary-value problem for a linear loaded integro-differential equation in an infinite three-dimensional domain. Chaos, Solitons \& Fractals, 140, p.110108. 
9. Zheng, Q., Xie, F. and Lin, W., 1999. Solution of two-dimensional Helmholtz equation by multipole theory method. Journal of electromagnetic waves and applications, 13(2), pp.205-220.

10. Thompson, L.L. and Pinsky, P.M., 1995. A Galerkin least-squares finite element method for the two-dimensional Helmholtz equation. International Journal for numerical methods in engineering, 38(3), pp.371-397.

11. Zhang, W. and Dai, Y., 2013. Finite-difference solution of the Helmholtz equation based on two domain decomposition algorithms. Journal of Applied Mathematics and Physics, 1(4), pp.18-24.

12. Samuel, M. and Thomas, A., 2010. On fractional Helmholtz equations. Fractional Calculus and Applied Analysis, 13(3), pp.295p-308p.

13. Gupta, P.K., Yildirim, A.H.M.E.T. and Rai, K.N., 2012. Application of He's homotopy perturbation method for multi-dimensional fractional Helmholtz equation. International Journal of Numerical Methods for Heat and Fluid Flow.

14. Baleanu, D., Jassim, H.K. and Al Qurashi, M., 2019. Solving Helmholtz equation with local fractional derivative operators. Fractal and Fractional, 3(3), p.43.

15. Abuasad, S., Moaddy, K. and Hashim, I., 2019. Analytical treatment of two-dimensional fractional Helmholtz equations. Journal of King Saud University-Science, 31(4), pp.659-666.

16. Prakash, A., Goyal, M. and Gupta, S., 2019. Numerical simulation of space-fractional Helmholtz equation arising in seismic wave propagation, imaging and inversion. Pramana, 93(2), p.28.

17. Prakash, A., Goyal, M. and Gupta, S., 2019. Numerical simulation of space-fractional Helmholtz equation arising in seismic wave propagation, imaging and inversion. Pramana, 93(2), p.28.

18. Momani, S. and Abuasad, S., 2006. Application of He's variational iteration method to Helmholtz equation. Chaos, Solitons \& Fractals, 27(5), pp.1119-1123.

19. Jarad, F. and Abdeljawad, T., 2018. A modifi ed Laplace transform for certain generalized fractional operators. Results in Nonlinear Analysis, 1(2), pp.88-98. 